\title{
Testing a Four-Dimensional Variational Data Assimilation Method Using an Improved Intermediate Coupled Model for ENSO Analysis and Prediction
}

\author{
Chuan $\mathrm{GAO}^{1,2}$, Xinrong $\mathrm{WU}^{3}$, and Rong-Hua ZHANG*1,4 \\ ${ }^{1}$ Key Laboratory of Ocean Circulation and Waves, Institute of Oceanology, Chinese Academy of Sciences, Qingdao 266071 \\ ${ }^{2}$ University of Chinese Academy of Sciences, Beijing 100029 \\ ${ }^{3}$ Key Laboratory of Marine Environmental Information Technology, State Oceanic Administration, \\ National Marine Data and Information Service, Tianjin 300000 \\ ${ }^{4}$ Laboratory for Ocean and Climate Dynamics, Qingdao National Laboratory for \\ Marine Science and Technology, Qingdao 266237
}

(Received 1 December 2015; revised 28 January 2016; accepted 19 February 2016)

\begin{abstract}
A four-dimensional variational (4D-Var) data assimilation method is implemented in an improved intermediate coupled model (ICM) of the tropical Pacific. A twin experiment is designed to evaluate the impact of the 4D-Var data assimilation algorithm on ENSO analysis and prediction based on the ICM. The model error is assumed to arise only from the parameter uncertainty. The "observation" of the SST anomaly, which is sampled from a "truth" model simulation that takes default parameter values and has Gaussian noise added, is directly assimilated into the assimilation model with its parameters set erroneously. Results show that 4D-Var effectively reduces the error of ENSO analysis and therefore improves the prediction skill of ENSO events compared with the non-assimilation case. These results provide a promising way for the ICM to achieve better real-time ENSO prediction.
\end{abstract}

Key words: Four-dimensional variational data assimilation, intermediate coupled model, twin experiment, ENSO prediction

Citation: Gao, C., X. R. Wu, and R.-H. Zhang, 2016: Testing a four-dimensional variational data assimilation method using an improved intermediate coupled model for ENSO analysis and prediction. Adv. Atmos. Sci., 33(7), 875-888, doi: $10.1007 / \mathrm{s} 00376-016-5249-1$.

\section{Introduction}

ENSO is the strongest interannual phenomenon in the tropical Pacific, directly inducing climate anomalies worldwide. Thus, accurately and effectively predicting ENSO events is of great significance to society. In recent decades, great advancements have been made in understanding ENSO and developing models for its real-time prediction (e.g., Bjerknes, 1969; Wyrtki, 1975; McCreary, 1983; Cane et al., 1986; Zhang et al., 2013). At present, various types of airsea coupled models have been developed, including intermediate coupled models (ICMs; e.g., Zebiak and Cane, 1987; Balmaseda et al., 1994; Zhang et al., 2003), hybrid coupled models (e.g., Neelin, 1990; Barnett et al., 1993; Zhang et al., 2015), and fully coupled general circulation models (e.g., Philander et al., 1992; Rosati et al., 1997). Currently, these coupled models enable us to make six-month to one-year

\footnotetext{
* Corresponding author: Rong-Hua ZHANG

Email: rzhang@qdio.ac.cn
}

real-time ENSO predictions in advance with reasonable success.

However, certain challenges still exit in the real-time prediction of ENSO events. For example, model biases cause ENSO simulations to depart far away from observations, making model state estimation and prediction inaccurate (Zhang et al., 2005c). In addition, high-quality ocean observations are very scarce, which results in uncertainties in ocean state estimation (Wang et al., 2000). Accordingly, difficulties emerge in providing accurate initial ocean conditions for ENSO prediction. Thus, it is essential to find a way to make model solutions coherent with observations by producing optimal initial conditions for predictions. To this end, data assimilation is an effective way to provide optimal initializations for ENSO analysis and prediction. However, a related issue is how to effectively use limited observations in data assimilation ( $\mathrm{Mu}$ et al., 2015). To achieve this, observing system experiments need to be performed to identify target observations with sensitive domains where data assimilation can be used effectively to improve prediction. Thus, 
an effective data assimilation system necessarily includes not only highly-quality data and a good data assimilation scheme, but also a method for these two aspects to be combined in a smart way, ultimately providing much more realistic initial analysis fields for predictions (Mu et al., 2015).

Various data assimilation algorithms have been used to initialize ENSO prediction. Originally, the nudging method, which directly forces the model solution to approximate observations, was used to force the modeled SST to reflect observed values to initialize ENSO prediction (e.g., Chen et al., 1995; Kirtman and Zebiak, 1997; Kumar et al., 2014; Zhu et al., 2015). Later, ensemble Kalman filters (Evensen, 1994) were introduced into ocean modeling, providing probabilistic forecasts of ENSO (e.g., Houtekamer and Mitchell, 1998; Zheng et al., 2006, 2009; Zhang et al., 2007; Tang et al., 2014; Wu et al., 2014). As an important branch of data assimilation, variational (three- and four-dimensional) methods (3D-Var and 4D-Var, respectively) are also widely used in ENSO analysis and prediction. For example, the 4D-Var data assimilation method pursues the analysis solutions by minimizing the distance between the model trajectory and observation time series [i.e., the so-called cost function (e.g., Tang and Hsieh, 2001; Zhang et al., 2001; Han et al., 2006, 2015; Peng and Xie, 2006; Zhang et al., 2015b)]. Compared with economic 3D-Var analysis (Derber and Rosati, 1989; Zhu et al., 2006), the 4D-Var data assimilation method is more dynamically and mathematically consistent (e.g., Dommenget and Stammer, 2004; Sugiura et al., 2008). For instance, Weaver et al. (2003) assimilated in situ temperature data into an OGCM by the 3D-Var and 4D-Var methods, and demonstrated that 4D-Var is more effective than 3D-Var in producing a consistent ocean state between model solutions and observations. Additionally, the 4D-Var method has been applied to ENSO prediction using various models and has achieved some success (Kleeman et al., 1995; Galanti et al., 2003; Dommenget and Stammer, 2004). The main difficulty in 4D-Var-based initialization of ENSO prediction is that the method requires the development of an adjoint model to compute the gradient of the cost function with respect to the control variables, which is very complicated and timeconsuming.

Our goal in this study is to implement the 4D-Var method to an improved intermediate coupled model (ICM) that was developed for ENSO studies (e.g., Zhang et al., 2003; Zhang et al., 2005b). The ICM used is a simplified coupled oceanatmosphere model with two statistical submodels for the temperature of subsurface water entrained into the mixed layer $\left(T_{\mathrm{e}}\right)$ and wind stress $(\tau) . T_{\mathrm{e}}$ is optimally calculated in terms of sea level (SL) anomalies using an EOF analysis technique. Wind stress anomalies are estimated based on an SVD analysis between SST anomalies and $\tau$ anomalies (Zhang et al., 2003, 2015). Therefore, the wind anomalies are represented as a response to SST, and the subsurface thermal effect on SST is parameterized by the ocean dynamical field. Although the ICM has been used for realistic predictions of ENSO (Zhang et al., 2013), it has not yet applied the 4D-Var method to initialize the real-time prediction. Since the 4D-
Var method is more dynamically and mathematically consistent in offering an initial ocean state for improving forecast accuracy, we specifically address the following question in this paper: Can the ENSO forecast skill generated by the ICM be increased by using the 4D-Var method?

Herein, we provide a detailed description of the incorporation of the 4D-Var data assimilation method into the aforementioned ICM, including the development of the associated tangent linear model and adjoint model. Based on the successful implementation of the 4D-Var data assimilation formulation into the ICM, we then report the preliminary results of a series of sensitivity experiments. Previously, Zheng et al. (2009) incorporated an ensemble Kalman filter method into the ICM and achieved improved ENSO prediction.

The paper is organized as follows: Section 2 describes the ICM and 4D-Var data assimilation method. The experimental setup is introduced in section 3 , and the assimilation impacts are analyzed in section 4. Finally, a conclusion and discussion are presented in section 5 .

\section{Methodology}

In this section, we briefly describe the ICM that has been routinely used to make ENSO predictions [see a summary of the model ENSO forecasts at the International Research Institute for Climate and Society (IRI) website: http://iri.columbia. edu/climate/ENSO/currentinfo/SST_table.html]; the real-time prediction results are posted on the IRI website every month, now referred to as the IOCAS (Institute of Oceanology/Chinese Academy of Sciences) ICM. Then, the 4D-Var data assimilation procedure is described, including its tangent linear model and adjoint model and the corresponding minimization processes.

\subsection{Description of the ICM}

The ICM consists of a dynamic ocean model, an SST anomaly model, and two statistical anomaly models for $T_{e}$ and $\tau$. The atmosphere component is a simple empirical statistical model for the $\tau$ anomaly, which depicts the response of $\tau$ to an SST field. It is constructed by the SVD method, based on historical data of the SST and $\tau$; symbolically, the relation between these two anomalous fields is expressed as $\tau=\alpha_{\tau} F_{\tau}\left(\mathrm{SST}_{\text {inter }}\right)$, in which $F_{\tau}$ is the relationship between $\tau$ and $\mathrm{SST}_{\text {inter }}$ derived using statistical methods from historical data, and $\alpha_{\tau}$ is a scalar parameter indicating the strength of wind forcing. The combined SVD method is used to obtain the covariance between the SST and zonal and meridional $\tau$ fields. The seasonality of interannual $\tau$ variability is taken into account with $12 \tau$ models constructed for each month. The $\tau$ field is then used to drive the ocean model.

The ocean component of the ICM includes a dynamical ocean model, an SST anomaly model, and a statistical model of $T_{e}$. The dynamical ocean model was developed by Keenlyside and Kleeman (2002), based on the McCreary (1981) baroclinic model. It includes linear and nonlinear parts. In the vertical direction, the modal decomposition approach is 
adopted to solve the linear part, which retains the first 10 baroclinic modes, whereas the higher 11 to 30 modes are represented only in the two surface layers. The nonlinear part is highly simplified and represented as the residual term in the momentum equation and is used to make a correction to the linear solutions that are ignored by the linear assumption that can be broken down in the equatorial region. It is worth noting that by introducing the horizontal stratification variation and partial nonlinear effects, the dynamic ocean model can simulate features of the actual equatorial current system well, such as the equatorial undercurrent and surface current and their seasonal variability (Keenlyside and Kleeman, 2002).

The SST anomaly model, which is embedded in the dynamical ocean model, describes the evolution of interannual temperature anomalies over the surface mixed layer. The time tendency of the SST anomaly is determined by its horizontal advection and diffusion terms, vertical advection and diffusion terms and thermal dissipation. The diagnostic analysis of the SST budget demonstrates that the vertical advection and diffusion terms (which are related to the $T_{\mathrm{e}}$ anomaly) are important in determining the variation in the SST anomaly. The SST anomaly model is equipped with a parameterization for $T_{\mathrm{e}}$ that is diagnosed by the sea level anomaly $\left(\mathrm{SL}_{\mathrm{inter}}\right)$ field based on an EOF. The relationship between the $T_{\mathrm{e}}$ anomaly $\left(T_{\mathrm{e}}^{\prime}\right)$ and $\mathrm{SL}_{\text {inter }}$ can be written as $T_{e}^{\prime}=\alpha_{\mathrm{Te}} F_{\mathrm{Te}}\left(\mathrm{SL}_{\mathrm{inter}}\right)$, in which $F_{\mathrm{Te}}$ is the relationship between $T_{\mathrm{e}}^{\prime}$ and $\mathrm{SL}_{\text {inter }}$ derived using statistical methods from historical data, and $\alpha_{\mathrm{Te}}$ is a scalar parameter introduced as the subsurface thermal forcing strength.

For each time step, the integration of the ICM can be sequentially implemented as follows (Zhang et al., 2005a): First, the SST anomaly equation is integrated to update the SST anomaly, which is used to calculate the $\tau$ anomaly based on the $\tau$ model. Second, the obtained $\tau$ anomaly field is used as the forcing to drive the dynamic ocean to update the SL, current fields in the mixed layer, and vertical velocity at the bottom of the mixed layer. Third, the $T_{\mathrm{e}}$ anomaly is calculated using the updated SL anomaly based on the $T_{\mathrm{e}}$ model, which is then used to simulate the vertical thermal effect in the SST anomaly equation. Repeating these processes can provide interannual variations of the oceanic and atmospheric wind fields. Further details regarding the ICM can be found in the study by Zhang and Gao (2015).

\subsection{The 4D-Var data assimilation method}

The 4D-Var method achieves the analysis solution of initial fields through minimizing the distance between the model trajectory and observation, which is constrained strictly by the model dynamical equations (Klinker et al., 2000).

In general, the governing equations of the ICM can be symbolically expressed as follows (Kalnay, 2003):

$$
\begin{aligned}
\frac{\partial \boldsymbol{X}}{\partial t} & =F(\boldsymbol{X}), \\
\left.\boldsymbol{X}\right|_{t_{0}} & =\boldsymbol{X}_{0},
\end{aligned}
$$

where $t$ is time and $t_{0}$ is the initial time; $\boldsymbol{X}$ is the vector of control variables, which includes SST, SL and horizontal ocean current velocities ( $U$ and $V$ ) in the ICM; $\boldsymbol{X}_{0}$ is the initial value of $\boldsymbol{X}$; and $\boldsymbol{F}$ is the nonlinear forward operator.

For the 4D-Var algorithm, the cost function can be formulated as (Kalnay, 2003)

$$
\begin{aligned}
J\left(\boldsymbol{X}_{0}\right)= & \frac{1}{2}\left[\boldsymbol{X}\left(t_{0}\right)-\boldsymbol{X}_{\mathrm{b}}\right]^{\mathrm{T}} \boldsymbol{B}^{-1}\left[\boldsymbol{X}\left(t_{0}\right)-\boldsymbol{X}_{\mathrm{b}}\right]+ \\
& \frac{1}{2} \sum_{i=1}^{N}\left\{\boldsymbol{H}\left[\boldsymbol{X}\left(t_{i}\right)\right]-\boldsymbol{Y}_{\mathrm{o}}\left(t_{i}\right)\right\}^{\mathrm{T}} \boldsymbol{R}^{-1}\left\{\boldsymbol{H}\left[\boldsymbol{X}\left(t_{i}\right)\right]-\boldsymbol{Y}_{\mathrm{o}}\left(t_{i}\right)\right\}(2)
\end{aligned}
$$

where the superscript " $T$ " represents the transpose of a matrix and subscripts "b" and "o" represent the background field and observation, respectively; $N$ indicates the number of integrations in the minimization time window; $\boldsymbol{Y}_{o}$ represents the observation; and $\boldsymbol{B}, \boldsymbol{R}$ and $\boldsymbol{H}$ represent the background error covariance matrix, the observation error covariance matrix and the observation operator, respectively. In this study, $\boldsymbol{B}$ and $\boldsymbol{R}$ are simply set as the identity matrix multiplied by the standard deviation of the observational error.

An optimization algorithm is needed to obtain the optimal solutions. The input arguments of an optimization algorithm include the initial guess and the number of control variables, the cost function and the gradient of the cost function with respect to the control variable. The computation of the gradient of the cost function involves the backward integration of the adjoint model. Mathematically, if we consider the adjoint model as an operator, the adjoint model is the transpose of the tangent linear model that is the linearization of the nonlinear forward model. Whether an optimization algorithm can correctly yield an analysis solution depends on the accuracy of the gradient. Thus, it is necessary to examine the accuracy of the gradient computed by the adjoint model. At this point, the tangent linear model is an effective tool to perform the abovementioned verification. In this section, we simply introduce the tangent linear model and the adjoint model of the ICM, as well as the optimization algorithm used in this study.

\subsubsection{The tangent linear model}

The tangent linear model results from the linearization of the original nonlinear model. The model is not directly involved in the 4D-Var data assimilation procedure, but it is helpful for developing the adjoint model and testing whether the adjoint model is correct.

The tangent linear model of the ICM can be expressed as (Kalnay, 2003)

$$
\begin{aligned}
\frac{\partial \boldsymbol{X}^{\prime}}{\partial t} & =\frac{\partial F(\boldsymbol{X})}{\partial \boldsymbol{X}} \boldsymbol{X}^{\prime}=\boldsymbol{M}(\boldsymbol{X}) \boldsymbol{X}^{\prime}, \\
\left.\boldsymbol{X}^{\prime}\right|_{t_{0}} & =\boldsymbol{X}_{0}^{\prime},
\end{aligned}
$$

where $\boldsymbol{X}^{\prime}$ is a small perturbation vector of $\boldsymbol{X}$ and $\boldsymbol{M}(\boldsymbol{X})=$ $\partial F(\boldsymbol{X}) / \partial \boldsymbol{X}$ is the tangent linear operator of $\boldsymbol{F}$, which is a firstorder approximation.

To verify whether the established tangent linear model of the ICM is correct, one can use a formula based on the firstorder approximation as follows (Navon et al., 1992):

$$
\mathrm{RV}=\frac{\left\|F\left(\boldsymbol{X}+\delta \boldsymbol{X}^{\prime}\right)-F(\boldsymbol{X})\right\|}{\delta\left\|\boldsymbol{M}\left(\boldsymbol{X}, \boldsymbol{X}^{\prime}\right)\right\|}=\mathbf{1}+O(\delta),
$$


where $\|\cdot\|$ is the L2-norm; $\delta$ is a small value ranging from 0 to 1 , and $O(\delta)$ is the high-order small perturbation. RV is the ratio of the differences between the ICM variable tendencies caused by a small perturbation in $\delta$ to the perturbation calculated by the tangent linear model, which ideally should approach 1 . The test results (double precision) of the tangent linear model in association with the 4D-Var method based on the ICM are shown in Table 1. As $\delta$ gradually decreases by one order of magnitude from $10^{-1}$ to $10^{-5}$, the value of RV consistently approaches 1 . It should also be noted that when $\delta$ is too small, e.g., with a decrease by one order of magnitude from $10^{-6}$ to $10^{-10}$, the value of RV conversely becomes slightly larger, which is the result of a truncation error. Thus, it is evident that the established tangent linear model of the ICM is correct.

\subsubsection{The adjoint model}

Generally, the adjoint model is an efficient solution for evaluating the gradient of the cost function with respect to high-dimensional control variables in the 4D-Var data assimilation method. The model is the transpose of the tangent linear model, i.e., it features the reverse of the temporal and spatial integration and other characteristics.

The equations of the adjoint model of the ICM can be written as follows (Kalnay, 2003):

$$
\begin{array}{r}
-\frac{\partial \boldsymbol{X}^{*}}{\partial t}=\left(\frac{\partial F(\boldsymbol{X})}{\partial \boldsymbol{X}}\right)^{\mathrm{T}} \boldsymbol{X}^{*}=\boldsymbol{M}^{\mathrm{T}} \boldsymbol{X}^{*}=\boldsymbol{M}^{*} \boldsymbol{X}^{*}, \\
\left.\boldsymbol{X}^{*}\right|_{t=N}=0,
\end{array}
$$

where $\boldsymbol{X}^{*}$ is the adjoint of $\boldsymbol{X}$ and $\boldsymbol{M}^{*}=(\partial F(\boldsymbol{X}) / \partial \boldsymbol{X})^{\mathrm{T}}=\boldsymbol{M}^{\mathrm{T}}$ is the adjoint of $\boldsymbol{M}$, which is the tangent linear model of the ICM. The gradient of the cost function is obtained by a backward integration of the adjoint model.

Based on the relationship between the tangent linear model and the adjoint model, one can verify the accuracy of the adjoint model using the following formula (Navon et al., 1992):

$$
\left\langle\boldsymbol{M} \boldsymbol{X}_{0}, \boldsymbol{M} \boldsymbol{X}_{0}\right\rangle=\left\langle\boldsymbol{M}^{*} \boldsymbol{M} \boldsymbol{X}_{0}, \boldsymbol{X}_{0}\right\rangle
$$

Table 1. Test results (double precision) obtained for the tangent linear model in association with the 4D-Var method based on the ICM. Here, $\delta$ is a small value gradually approaching 0 , and $\mathrm{RV}$ is the ratio of the differences between the ICM variable tendencies caused by a small perturbation in $\delta$ to the perturbation calculated by the tangent linear model, which ideally should approach 1.

\begin{tabular}{cc}
\hline$\delta$ & $\mathrm{RV}$ \\
\hline $10^{-1}$ & 0.9969131317710270 \\
$10^{-2}$ & 0.9996664164813890 \\
$10^{-3}$ & 0.9999692317079850 \\
$10^{-4}$ & 0.9999969328883560 \\
$10^{-5}$ & 0.9999996029827230 \\
$10^{-6}$ & 0.9999993932446230 \\
$10^{-7}$ & 1.0000248186382400 \\
$10^{-8}$ & 1.0002195754411600 \\
$10^{-9}$ & 1.0005791264619800 \\
$10^{-10}$ & 1.0024624889518900 \\
\hline
\end{tabular}

where $\langle$,$\rangle represents the inner product between the two vec-$ tors. For the LHS of Eq. (6), the tangent linear model is integrated forward using the initial condition $\boldsymbol{X}_{0}$ to obtain $\boldsymbol{M} \boldsymbol{X}_{0}$, which is then used to compute its own inner product. For the RHS of Eq. (6), the adjoint model is integrated from the initial condition $\boldsymbol{M} \boldsymbol{X}_{0}$ to obtain $\boldsymbol{M}^{*} \boldsymbol{M} \boldsymbol{X}_{0}$, which is used to compute the inner product with the initial condition $\boldsymbol{X}_{0}$. Then, how one inner equals the other can be checked with a given precision.

Following the above-described approach, we perform a set of sensitivity experiments to demonstrate how the accuracy of the adjoint model of the ICM is affected by the length of the assimilation time window in the 4D-Var data assimilation process. Table 2 presents the test results for the experiments, obtained using different assimilation time windows (days). The results show that at least the first 10 valid digits of $\left\langle\boldsymbol{M} \boldsymbol{X}_{0}, \boldsymbol{M} \boldsymbol{X}_{0}\right\rangle$ are equal to those of $\left\langle\boldsymbol{M}^{*} \boldsymbol{M} \boldsymbol{X}_{0}, \boldsymbol{X}_{0}\right\rangle$ when using different assimilation time windows, indicating that the adjoint model is accurate. Additionally, as the length of the assimilation window becomes longer from 4 days to 28 days, the equal valid digits become shorter from 12 to 10 ; this is because the nonlinearity becomes stronger as the length of the assimilation window becomes longer. Note that the experimental settings in the tangent linear model and adjoint model must remain the same as in the original nonlinear model, including the resolution, time step, physical processes and simplified dynamics.

\subsubsection{The minimization procedure}

After the adjoint model of the ICM is properly constructed, a minimization algorithm is used to find the 4D-Var analysis solution. First, the ICM model is integrated forward from an initial guess of $\boldsymbol{X}_{0}$ to obtain the cost function $J$. Second, the ICM is integrated backward with the adjoint model to obtain the gradient of $J$ with respect to $\boldsymbol{X}_{0}$. Third, the Limited-Memory BFGS (L-BFGS) algorithm (Liu and Nocedal, 1989) is used to minimize the cost function to obtain the analysis solution of $\boldsymbol{X}_{0}$ (Zou et al., 1993). The L-BFGS algorithm is an improved version of the BFGS algorithm, which is a quasi-Newton algorithm. The L-BFGS requires

Table 2. Test results (double precision) for the adjoint model of the ICM in the 4D-Var data assimilation process, obtained using different assimilation time windows (days). Here, $\boldsymbol{M}$ is the tangent linear model operator; $\boldsymbol{M}^{*}$ is the adjoint model operator; and $\boldsymbol{X}_{0}$ is the initial condition. The tangent linear model is integrated forward using the initial condition $\boldsymbol{X}_{0}$ to obtain $\boldsymbol{M} \boldsymbol{X}_{0}$, which is saved as $\left\langle\boldsymbol{M} \boldsymbol{X}_{0}, \boldsymbol{M} \boldsymbol{X}_{0}\right\rangle$, and the adjoint model is integrated backward from the initial condition $\boldsymbol{M} \boldsymbol{X}_{0}$ to obtain $\boldsymbol{M}^{*} \boldsymbol{M} \boldsymbol{X}_{0}$, which is saved as $\left\langle\boldsymbol{M}^{*} \boldsymbol{M} \boldsymbol{X}_{0}, \boldsymbol{X}_{0}\right\rangle$. The difference between $\left\langle\boldsymbol{M} \boldsymbol{X}_{0}, \boldsymbol{M} \boldsymbol{X}_{0}\right\rangle$ and $\left\langle\boldsymbol{M}^{*} \boldsymbol{M} \boldsymbol{X}_{0}, \boldsymbol{X}_{0}\right\rangle$ is an indicator of the accuracy of the $4 \mathrm{D}$-Var data assimilation process.

\begin{tabular}{ccc}
\hline Time window & $\left\langle\boldsymbol{M} \boldsymbol{X}_{0}, \boldsymbol{M} \boldsymbol{X}_{0}\right\rangle$ & $\left\langle\boldsymbol{M}^{*} \boldsymbol{M} \boldsymbol{X}_{0}, \boldsymbol{X}_{0}\right\rangle$ \\
\hline 4 Days & 38243.9322968130 & 38243.9322965545 \\
7 Days & 101439.163365451 & 101439.163359537 \\
14 Days & 306869.173465571 & 306869.173491971 \\
28 Days & 789400.566949510 & 789400.566192024 \\
\hline
\end{tabular}


four input arguments: an initial guess for the value of $\boldsymbol{X}_{0}$, the dimension of $\boldsymbol{X}_{0}$, the cost function $J$, and the gradient of the cost function with respect to $\boldsymbol{X}_{0}$.

An example of the convergence of the cost function with respect to the iteration number is shown in Fig. 1. The figure shows that the cost function rapidly reaches equilibrium after four iterations. Thus, the 4D-Var based on the ICM is efficient and reliable. To save on computational cost, we set the maximum value of the iteration number to 20 , which is sufficient to satisfy the convergence of the cost function. At this point, the 4D-Var data assimilation based on the ICM has been established.

\section{Assimilation experiments}

To partly reflect reality, we design a biased twin experiment (Zhang et al., 2014; Zhang et al., 2015a; Wu et al., 2016) to test the 4D-Var method, and report the results in this section. The twin experiment includes the observing network, model error and the assimilation schemes. Note that the model settings are the same for all data assimilation and prediction experiments, which prevents the "initial shock" that can result from the inconsistency of the coupled model physics and initial conditions in the transition from the assimilation phase to the prediction phase using the ICM (Keenlyside et al., 2005).

\subsection{Observing network and model error}

We assume that only the SST anomaly is observed and sampled once a day from the "truth" model that takes the default values of model parameters. The observed position of the SST anomaly is assumed to be the same as that of the model grids. To simulate the observational error, Gaussian noise with a mean and standard deviation of 0 and 0.2 is added to the sampled "truth" daily SST anomaly.

For the assimilation model, we assume that the model error only arises from parameter perturbations to roughly mimic the model error in the real situation. The default values of three model parameters are modified: the coupling

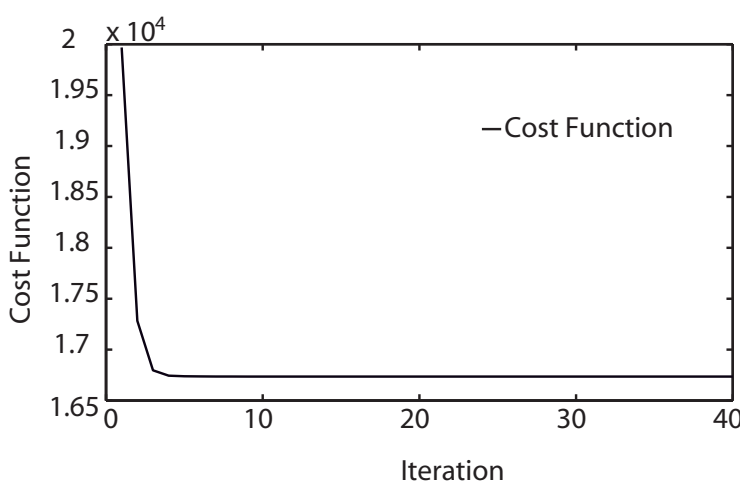

Fig. 1. Variation in the cost function with respect to the iteration number. Here, the cost function is defined as the sum of the background error term and observational error term, which decreases rapidly and converges to a constant value. coefficient between the SST anomaly and $\tau$ anomaly, $\alpha_{\tau}$, which is varied from 1.03 to $1.03 \times 1.01$; the vertical diffusivity coefficient, $K_{v}$, which is varied from $1.0 \times 10^{-3}$ to $1.0 \times$ $10^{-3} \times 0.95$; and the thermal damping coefficient, $\lambda$, which is varied from $1 /(100 \times 86400)$ to $1 /(100 \times 86400) \times 1.01$. The modifications of these parameters cause the trajectories of the assimilation model to depart from those of the "truth" model. However, the basic ENSO features simulated by the assimilation model, such as the spatiotemporal structure and the amplitude, remain unchanged. Starting from the same initial conditions, these two simulations are respectively conducted for 200 model years. Note that each model calendar month is assumed to have 30 days in this study. Figure 2a shows the time series of the Niño3.4 indices for the "truth" model and the assimilation model in the first 100-year simulations. It is clear that both model simulations can simulate the prominent ENSO features. Note that the stochastic forcing of the atmospheric wind field is not included in the ICM; thus the ENSO events depicted by the ICM are quite regular (Zhang et al., 2008; Zhang and Gao, 2015). In addition, even though the two model simulations start from the same initial conditions, the simulated Niño3.4 indices gradually depart from each other. To detect the significant periods of ENSO events produced by the two simulations, we perform a power spectrum analysis of the Niño3.4 indices with the total 200-year outputs (Fig. 2b). The results indicate that both model simulations have a $2-7$-year period that passes the $95 \%$ confidence level. The "truth" model has a dominant period of 3.81 years, whereas the assimilation model has a dominant period of 3.92 years.

\subsection{Assimilation designs}

In this study, three experiments are conducted to evaluate the 4D-Var data assimilation method based on the ICM: Expt. 1 is the control experiment of the "truth" model, used to produce the "observation" field; Expt. 2 is the 4D-Var assimilation experiment, which provides the optimal initial conditions by assimilating the "observation" of the SST anomaly; and Expt. 3 is the non-assimilation experiment of the assimilation model. The simulation period comprises 20 years from model time 2080/01/01 to 2099/12/30.

Figure 3 is a schematic diagram illustrating the experimental configuration for Expt. 2. From the initial condition (restart0) at model time 2000/01/01 (which is represented as 1 January 2000, in the model time), both the "truth" model and the assimilation model are integrated for 80 years. It is clear that the two simulations diverge from each other (see Fig. 2a). Then, the "truth" model is further integrated for 20 years from restart1 at model time 2080/01/01 to generate the "observations". The assimilation model is then integrated forward by assimilating "observations" from restart2 at model time 2080/01/01. In this 4D-Var data assimilation process, the "observed" SST anomaly is assimilated into the assimilation model at the first step of every day. The length of the minimization time window in this 4D-Var is determined by trial and error. In this study, considering the nonlinearity effect and the computational efficiency, the minimization 
(a) Nino3.4 Index
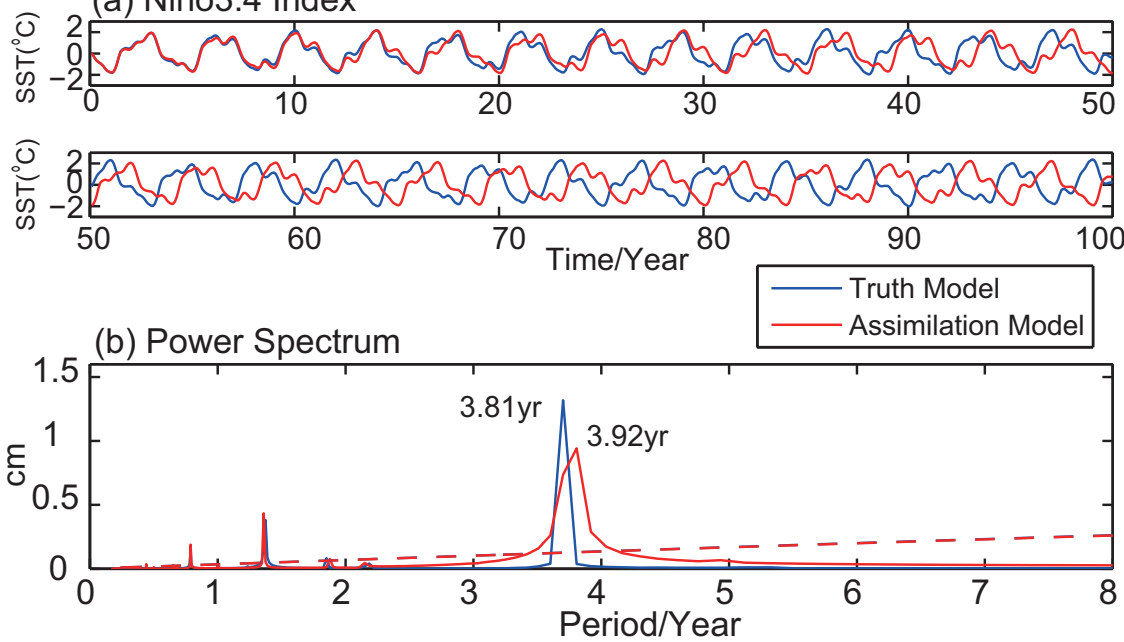

Fig. 2. (a) Time series of the Niño3.4 indices for the "truth" model (blue) and the assimilation model (red) in the first 100-year simulations. (b) Power spectrum analysis of the Niño3.4 indices during the first 200-year simulations for the "truth" model (blue) and the assimilation model (red), with the $95 \%$ confidence level indicated by the dashed curve.

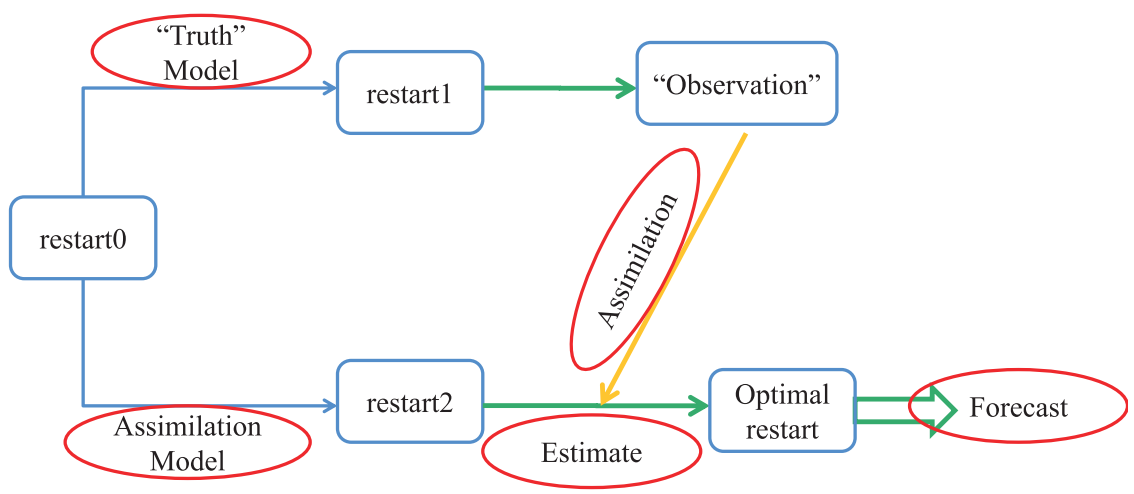

Fig. 3. Schematic diagram illustrating the process of the 4D-Var data assimilation. The "truth" model and the assimilation model are integrated from the same initial condition (restart0), and the models gradually diverge from each other. Then, the "truth" model is integrated for 20 years from restart1 to sample "observations", which are assimilated into the assimilation model with 4D-Var to obtain the optimal initial condition for ENSO prediction.

time window is set to 15 days in length. For example, starting from restart 2 at model time 2080/01/01, the assimilation model is subject to assimilating daily "observations" of the SST anomaly within a 15-day window to obtain the optimal initial condition at model time 2080/01/01. The assimilation model is then integrated with the optimal initial condition until model time 2080/01/16 to enter the next data assimilation cycle. Thus, each month has two data assimilation cycles.

The key measure for assessing assimilation quality is the prior RMSE, which is defined as

$$
\mathrm{RMSE}=\sqrt{\frac{1}{G} \sum_{i=1}^{G}\left(\boldsymbol{X}_{i}-\boldsymbol{X}_{\mathrm{truth}_{i}}\right)^{2},}
$$

where $\boldsymbol{X}$ is the control vector; $\boldsymbol{X}_{\text {truth }}$ is the corresponding "truth" vector obtained from Expt. $1 ; i$ is the grid index; and
$G$ is the total number of model grids. When the RMSE falls to a value that changes only slightly, the assimilation method is considered to have a converged solution. The assimilation period is chosen to have 20 model years. The results show that the spin-up period of the state estimation is approximately 2 years.

\section{Assessing the impacts of data assimilation}

The principles of the twin experiment are introduced in section 3 , where the assimilation model is assimilated with the "observation" field to retrieve the analysis solution. To assess the success of this 4D-Var data assimilation method based on the ICM, the focus will be on ENSO phenomena when performing the twin experiment. In this section, the ef- 


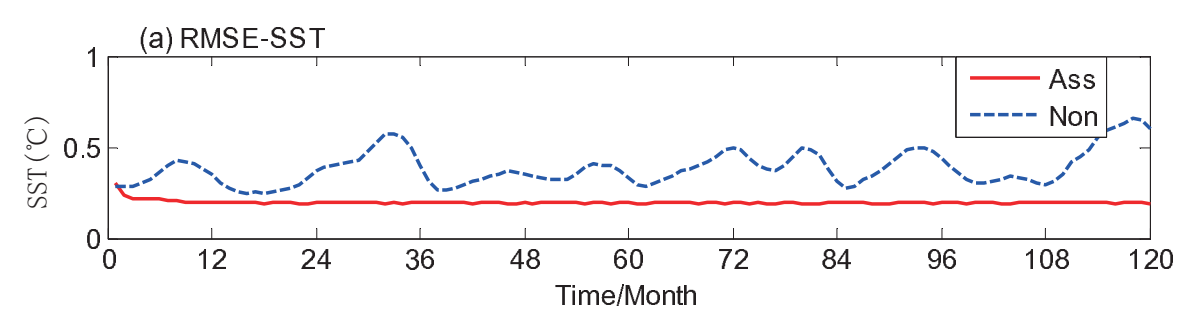

(b) RMSE-Taux

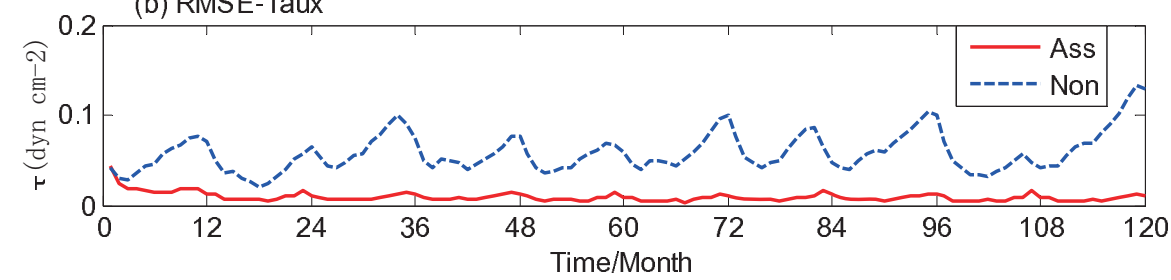

(c) RMSE-SL

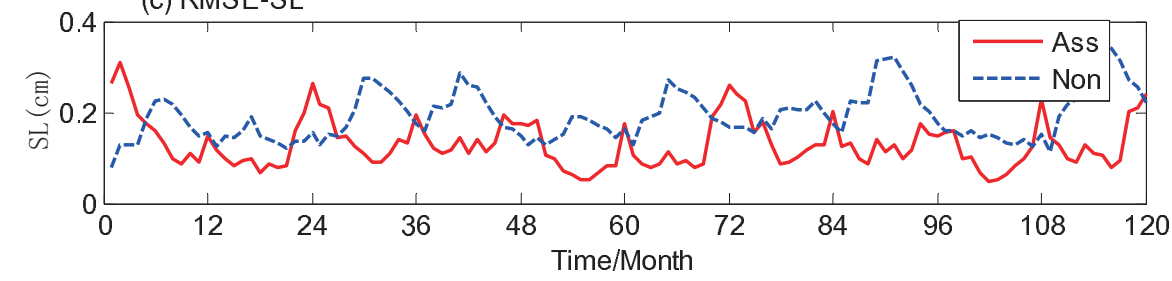

Fig. 4. Time series of RMSEs for prior anomalies of (a) SST (units: ${ }^{\circ} \mathrm{C}$ ), (b) zonal $\tau$ (units: dyn $\mathrm{cm}^{-2}$ ) and (c) SL (units: $\mathrm{cm}$ ) over the full tropical Pacific region $\left(30^{\circ} \mathrm{N}-30^{\circ} \mathrm{S}, 124^{\circ} \mathrm{E}-78^{\circ} \mathrm{W}\right)$. Here, the RMSE is calculated on the first day of each month in the first 10-year simulations for the assimilation (red) and non-assimilation (blue) experiments.

fect of assimilation on ENSO analysis is demonstrated first, and that on ENSO prediction is then discussed.

\subsection{Impact on ENSO analysis}

In this section, we first check the time series of the RMSEs of several key variables. Figure 4 shows the time series of RMSEs for prior anomalies of SST, zonal $\tau$ and SL over the full tropical Pacific region $\left(30^{\circ} \mathrm{N}-30^{\circ} \mathrm{S}, 124^{\circ} \mathrm{E}-78^{\circ} \mathrm{W}\right)$ for Expt. 2 and Expt. 3. Being directly assimilated, the RMSE of the SST anomaly (Fig. 4a) is rapidly reduced. Because the $\tau$ anomaly is directly diagnosed from the SST anomaly using its SVD model, it is the first beneficiary of the assimilation of the SST anomaly. The RMSE of the zonal wind anomaly (Fig. 4b) is rapidly reduced, similar to the RMSE of the SST anomaly. In contrast, the SL anomaly is indirectly affected by the SST anomaly assimilation, causing most RMSEs of the SL anomaly produced in Expt. 2 to be smaller than those produced in Expt. 3 (Fig. 4c). All the RMSEs indicate that assimilating the "observations" of the SST anomaly into the ICM by 4D-Var can improve the model state estimate, thus being able to provide optimal initial conditions.

Secondly, we examine the spatial RMSEs for Expt. 2 and Expt. 3. Figure 5 plots the spatial distributions of RMSEs for prior anomalies of SST, zonal and meridional $\tau$, SL and $T_{e}$ for Expt. 2 and Expt. 3. The RMSE here for each grid is calculated as follows:

$$
\mathrm{RMSE}_{i, j}=\sqrt{\frac{1}{N} \sum_{t=1}^{N}\left(\boldsymbol{X}_{i, j, t}-\boldsymbol{X}_{\text {truth }_{i, j, t}}\right)^{2}},
$$

where $\boldsymbol{X}$ represents the vector of the anomaly variables, including SST, zonal and meridional $\tau$, SL and $T_{e} ; \boldsymbol{X}_{\text {truth }}$ is the corresponding "truth" value of $\boldsymbol{X} ; i$ and $j$ represent the $(i, j)$ grid; $t$ is the time index; and $N$ is the total number of analysis times. The RMSEs of all variables for Expt. 2 (Figs. 5a-e) are much smaller than those for Expt. 3 (Figs. 5f-j), but the spatial patterns are quite similar. For the SST anomaly, both maximum RMSEs $\left[0.15^{\circ} \mathrm{C}\right.$ for Expt. 2 (Fig. 5a) and $0.5^{\circ} \mathrm{C}$ for Expt. 3 (Fig. 5f)] are centered in the eastern and central equatorial Pacific. For the zonal $\tau$ anomaly, the maximum RMSEs are located in the central equatorial Pacific, with values of $0.027 \mathrm{dyn}^{-2}$ and $0.16 \mathrm{dyn}^{-2}$ for Expt. 2 (Fig. 5b) and Expt. 3 (Fig. 5g), respectively. Similar results are obtained for the meridional $\tau$ anomaly (Figs. 5c and h), SL anomaly (Figs. 5d and i) and $T_{e}$ anomaly (Figs. 5e and j). Generally speaking, the differences in the RMSEs of the SST and $\tau$ (zonal and meridional components) anomalies between Expt. 2 and Expt. 3 are much larger than those of the $T_{e}$ and SL anomalies. The reason is the fact that only the "observations" of the SST anomaly are assimilated in Expt. 2 and $\tau$ anomalies are directly calculated from the SST anomaly field using the $\tau$ model. Thus, the assimilation process has a direct effect on the SST anomaly field and thereby on the $\tau$ anomaly field. In contrast, the SL and $T_{e}$ fields are indirectly impacted by the SST anomaly assimilation through the model physical processes. In general, the RMSEs produced by Expt. 2 are slightly smaller than those produced by Expt. 3. These results demonstrate that the 4D-Var method can effectively reduce the error in the initial conditions, thereby leading to 
(a) SST Rmse

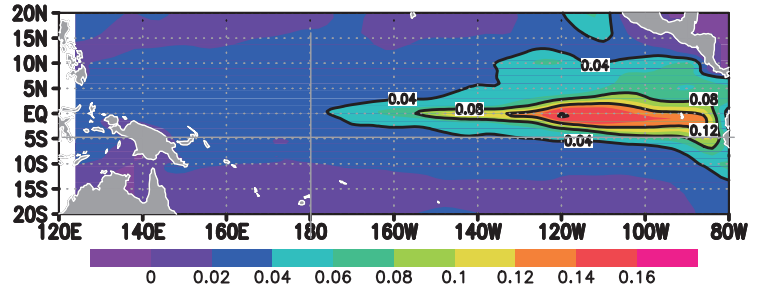

(b) Taux Rmse

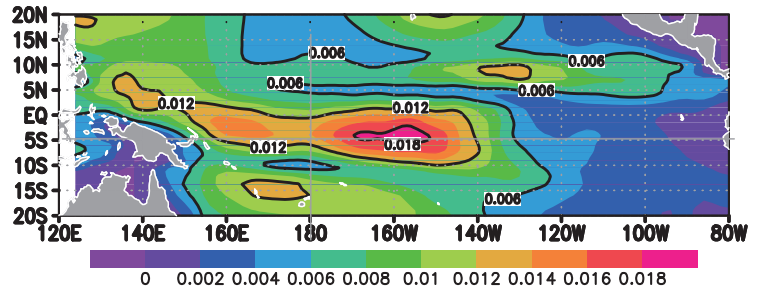

(c) Tauy Rmse

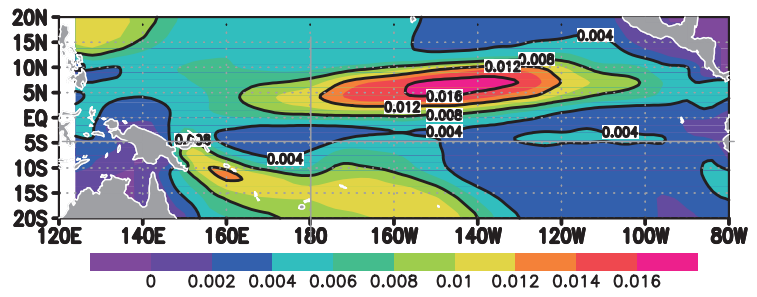

(d) SL Rmse

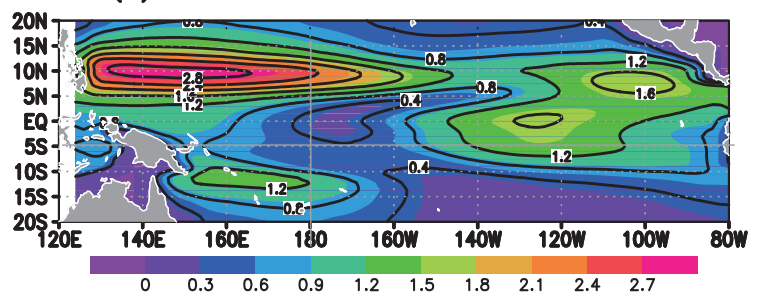

(e) Te Rmse

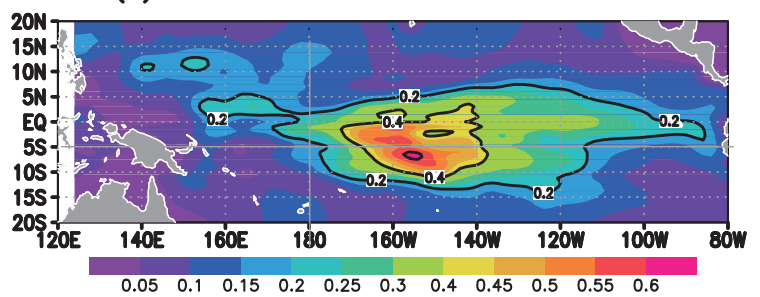

(f) SST Rmse

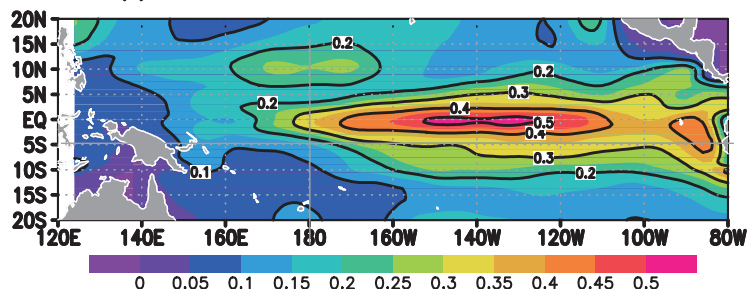

(g) Taux Rmse

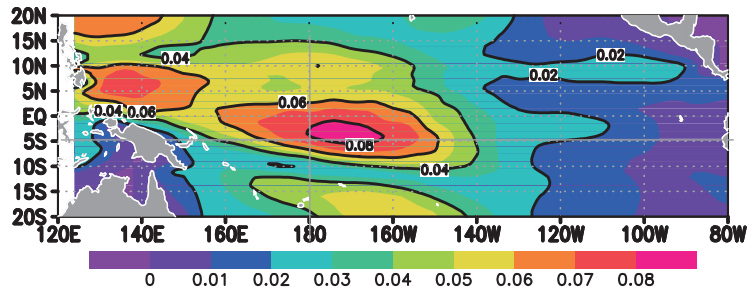

(h) Tauy Rmse

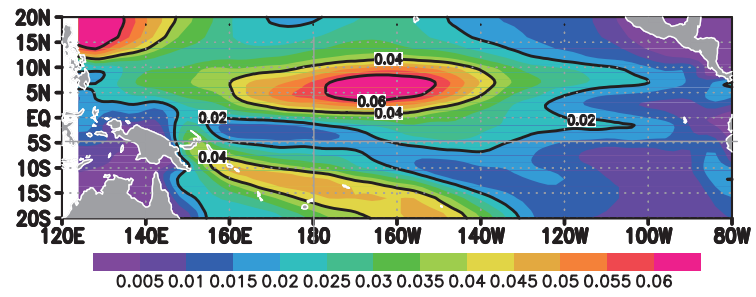

(i) SL Rmse

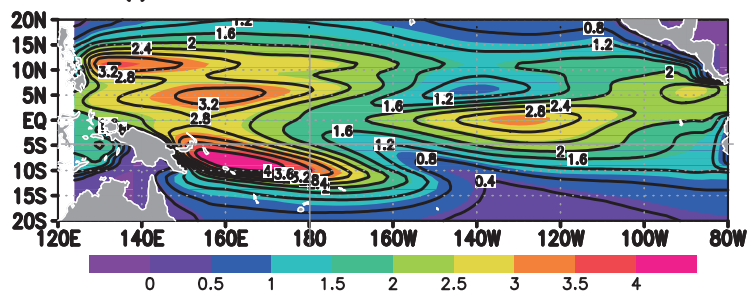

(j) Te Rmse

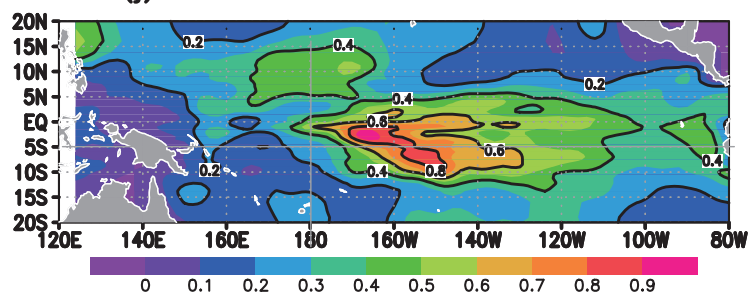

Fig. 5. Spatial distributions of RMSEs for prior anomalies of (a, f) SST (units: ${ }^{\circ} \mathrm{C}$ ), (b, g) zonal $\tau$ (units: dyn $\mathrm{cm}^{-2}$ ), $(\mathrm{c}, \mathrm{h}$ ) meridional $\tau$ (units: dyn $\mathrm{cm}^{-2}$ ), (d, i) SL (units: $\mathrm{cm}$ ), and $(\mathrm{e}, \mathrm{j}) T_{e}$ (units: ${ }^{\circ} \mathrm{C}$ ). Here, the RMSE is calculated from results obtained for the first 20-year simulations for the assimilation (left panels) and non-assimilation (right panels) experiments.

more accurate state estimations for ENSO events.

Thirdly, we check the temporal evolution of the SST and $\tau$ anomalies. Figure 6 shows the longitude-time sections of the SST anomalies along the equator during the first 12-year simulations (model time from 2080/01/01 to 2091/12/30) for the "truth" fields, Expt. 2 and Expt. 3. It can be seen that the ENSO period, spatial structure and phase transition are well represented in the ICM. Excluding the spin-up period of 4DVar, Expt. 2 (Fig. 6b) can retain nearly the same variability of the SST anomaly as in the "truth" simulation (Fig. 6a). For Expt. 3 (Fig. 6c), the biases arise from the initial conditions and the three model parameter perturbations cause the modeled SST anomaly to differ greatly from the "truth" field. For example, the amplitude of the modeled SST anomaly exhibits 


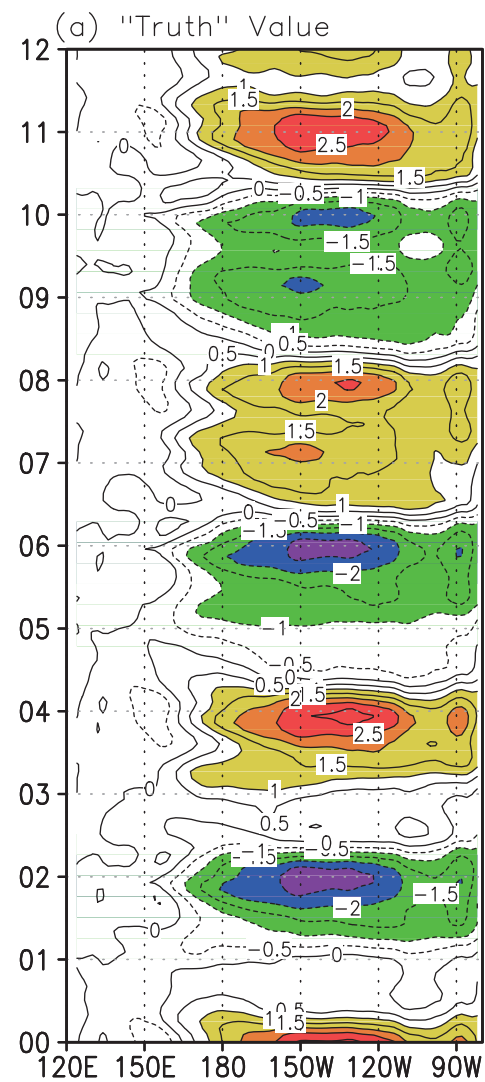

Anomalies along the equator
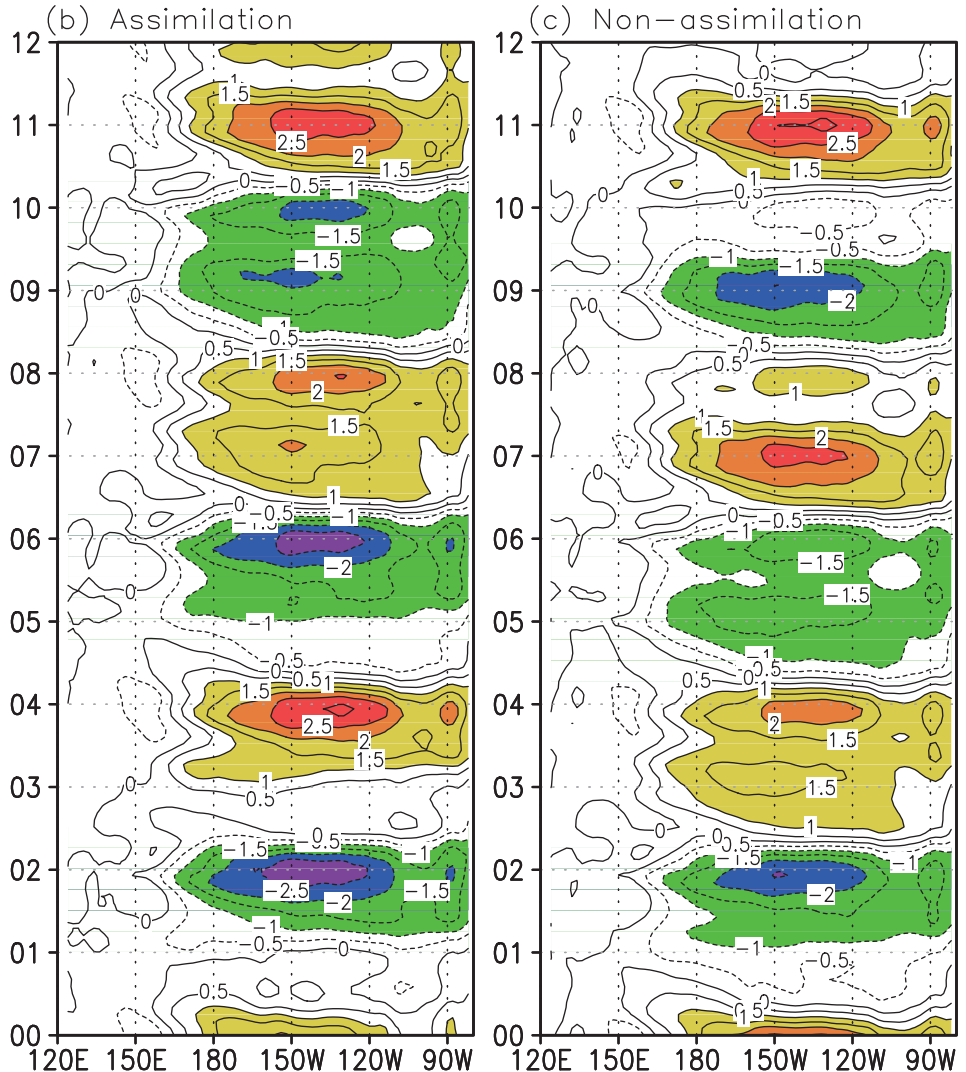

Fig. 6. Longitude-time sections of SST anomalies along the equator for the (a) "truth" value, (b) assimilation experiment and (c) non-assimilation experiment during the first 12-year simulations (model time period of 2080/01/01 to 2091/12/30). Contour interval: $0.5^{\circ} \mathrm{C}$.

some bias, especially in the eastern Pacific. Additionally, the phase transition time of the SST anomaly also differs from the "truth" value. Similar to Fig. 6, Fig. 7 illustrates the longitude-time sections of zonal $\tau$ anomalies along the equator. The spatiotemporal structure and amplitude of the zonal $\tau$ anomaly produced by Expt. 2 (Fig. 7b) are much more consistent with the "truth" field than those produced by Expt. 3 (Fig. 7c).

The ocean subsurface fields play an important role in the development of the ENSO events. To capture the ENSO events, it is necessary to adequately depict the $T_{e}$ field. Figure 8 shows the longitude-time sections of $T_{e}$ anomalies along the equator for the "truth" fields, Expt. 2 and Expt. 3. Through the model adjustment achieved by assimilating "observations" of the SST anomaly, the spatiotemporal evolution of $T_{e}$ produced by Expt. 2 (Fig. 7b) is in good agreement with the "truth" field (Fig. 7a) compared with that produced by Expt. 3 (Fig. 7c).

Finally, we check the analysis quality of ENSO produced by 4D-Var by taking the Niño3.4 index as the key parameter of ENSO. Figure 9a shows the time series of the Niño3.4 indices during the first 10-year simulations for the "truth" value, Expt. 2 and Expt. 3. It can be seen that Expt. 2 (red dashed) can keep tracking the "truth" value (green dotted) very well, whereas Expt. 3 (blue dashed) shows some deviation. For clarity, the time series of the absolute errors of the Niño3.4 indices in Expt. 2 and Expt. 3 are presented in Fig. 9b. It is evident that the absolute error produced by Expt. 2 is much smaller than that produced by Expt. 3. Furthermore, the absolute error produced by Expt. 3 gradually becomes larger (even reaching approximately $1.2^{\circ} \mathrm{C}$ ) due to the existence of model error. This finding again demonstrates that the 4D-var data assimilation can recover ENSO conditions well. Thus, the high level of agreement between the assimilation results and the "truth" value can provide a better initialization for ENSO prediction.

\subsection{Impact on ENSO prediction}

In general, a better prediction of ENSO events is a strict test for model simulation and analysis through data assimilation. Therefore, improved prediction accuracy is an important indicator for assessing the quality of the 4D-Var data assimilation approach. Based on state estimation with a 2-year spin-up period, we perform an array of 1-year forecast experiments starting from the analysis solutions on the first day in each month between the model time 2082/01/01 and 2099/12/01. Thus, there are $18 \times 12=216$ forecast experiments in total, which are used to perform the statistical 


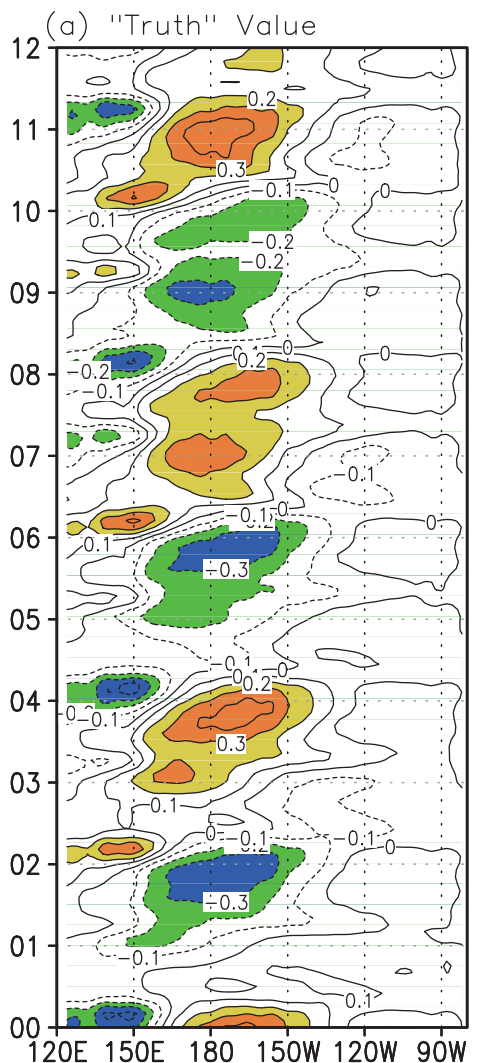

Anomalies along the equator
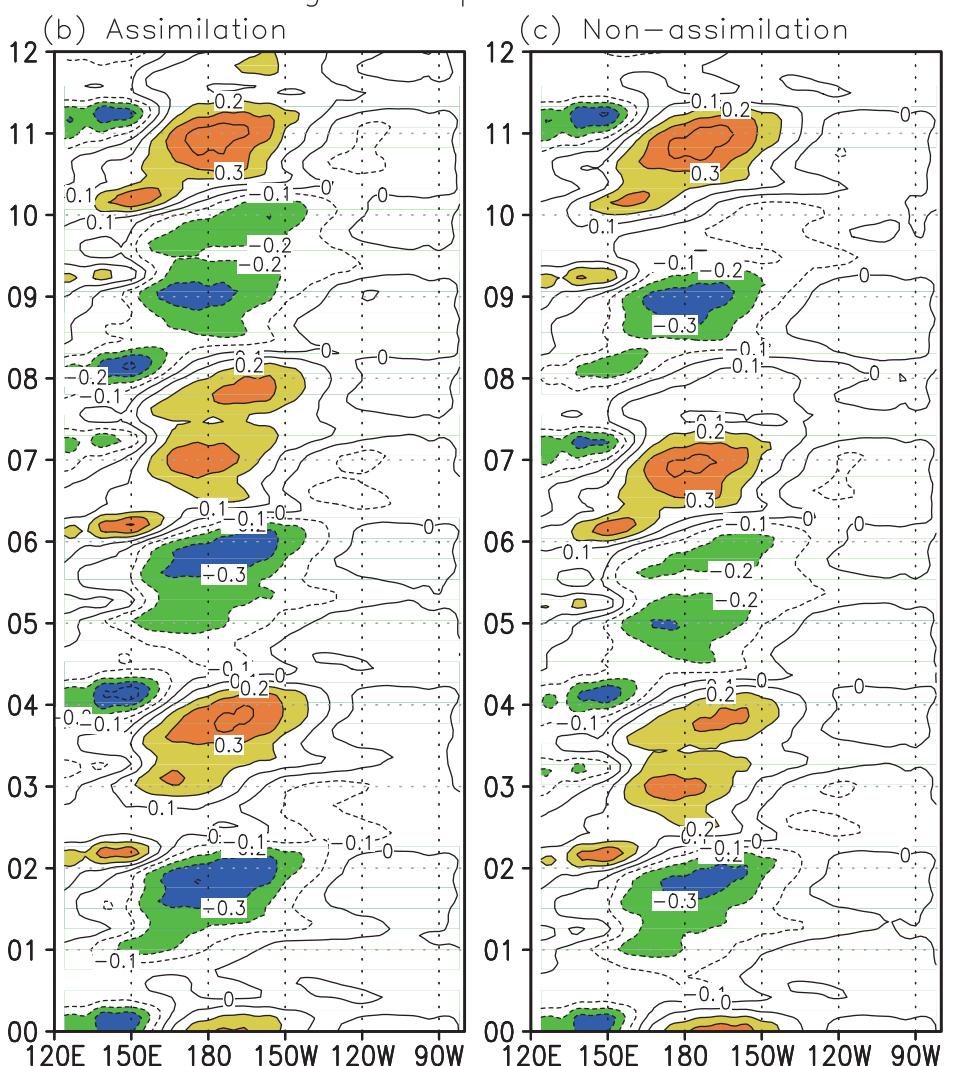

Fig. 7. As in Fig. 6 but for zonal wind anomalies. Contour interval: $0.1 \mathrm{dyn} \mathrm{cm}^{-2} .1 \mathrm{dyn} \mathrm{cm}{ }^{-2}=0.1 \mathrm{~N} \mathrm{~m}^{-2}$

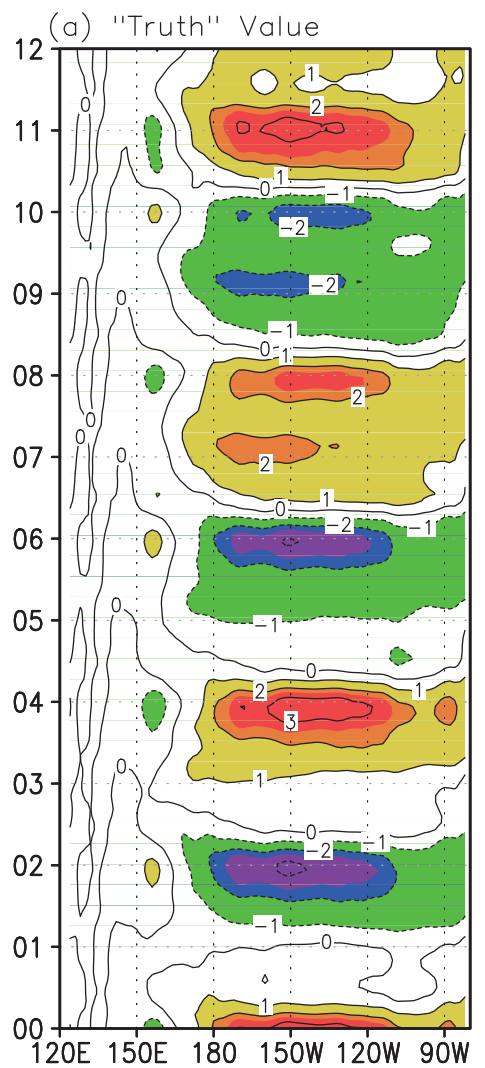
Anomalies along the equator
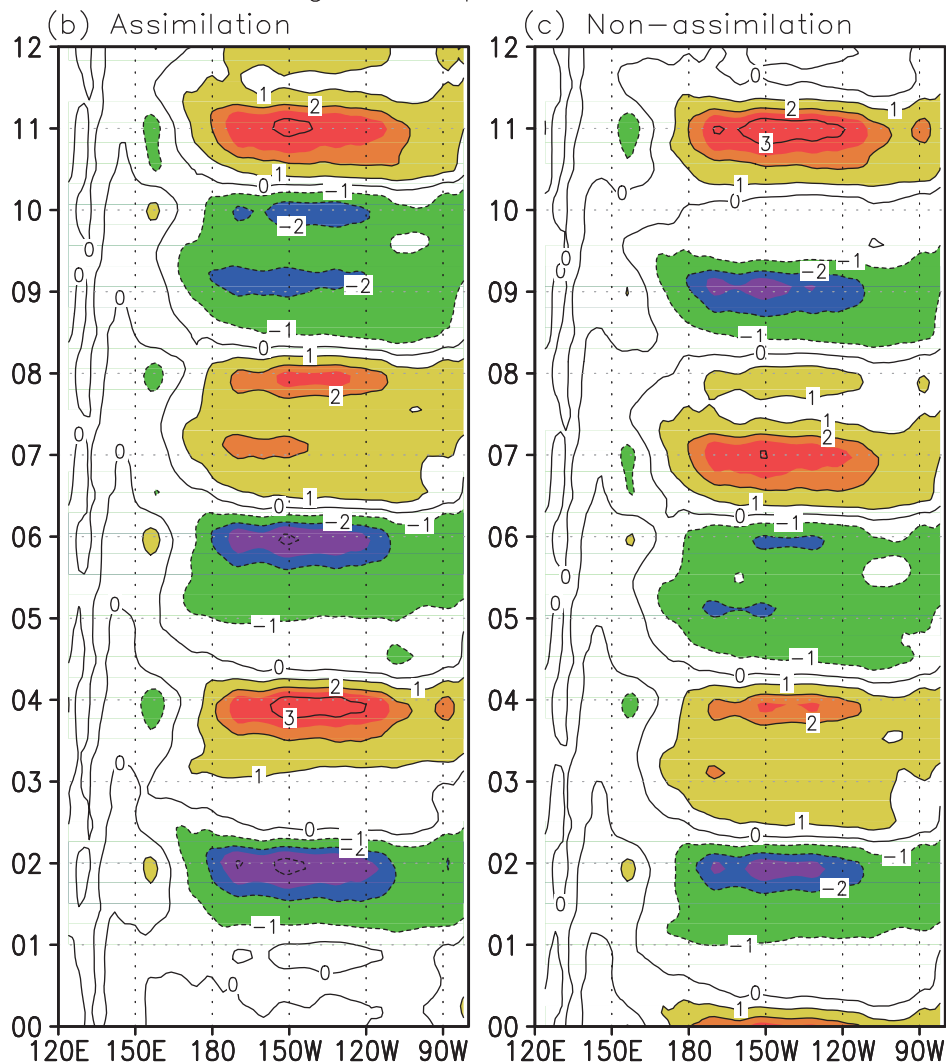

Fig. 8. As in Fig. 6 but for $T_{e}$ anomalies. Contour interval: $1^{\circ} \mathrm{C}$. 
(a) Nino3.4 Index
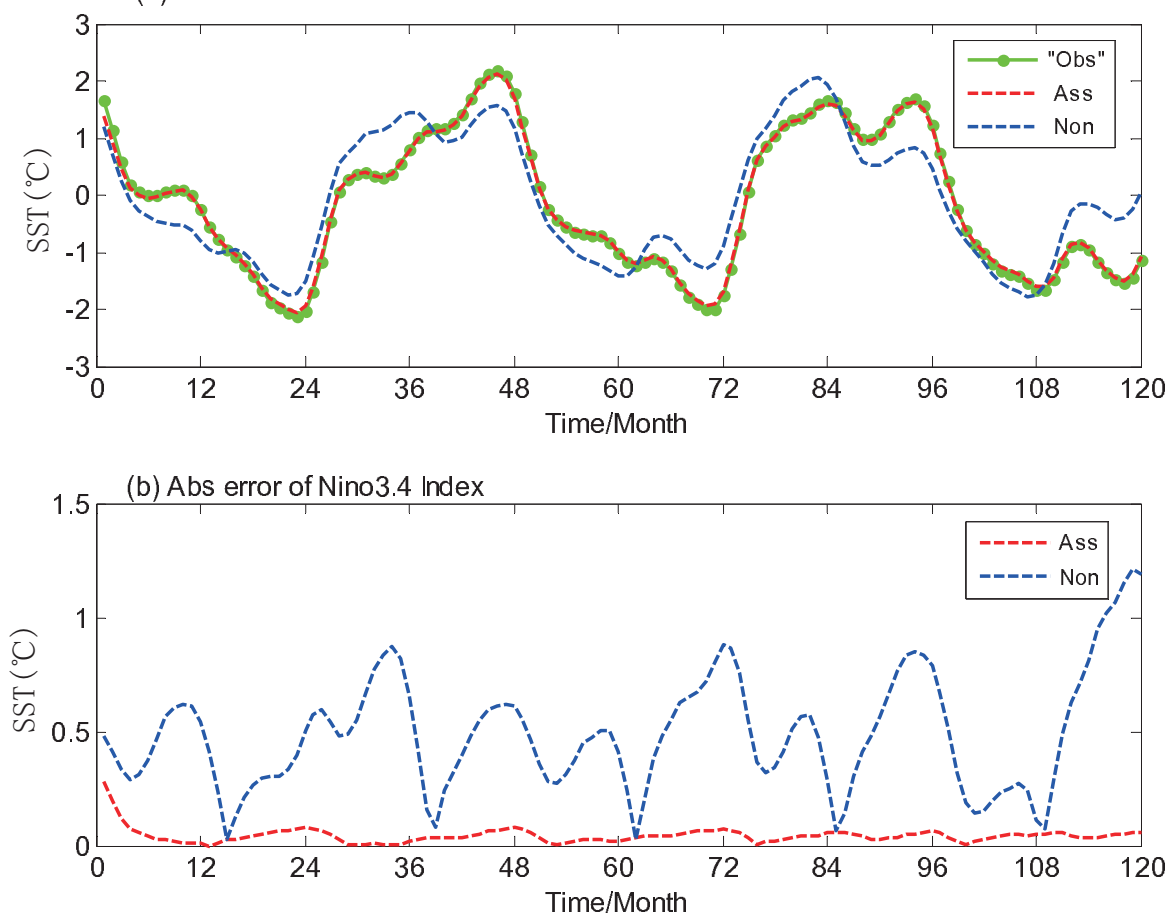

Fig. 9. (a) Time series of the Niño3.4 indices (units: ${ }^{\circ} \mathrm{C}$ ) for the "truth" value (green), assimilation experiment (red) and non-assimilation experiment (blue) during the first 10-year simulations. (b) Time series of the absolute errors of the Niño3.4 indices (unit: ${ }^{\circ}$ ) for the assimilation (red) and non-assimilation (blue) experiment during the first 10-year simulations.

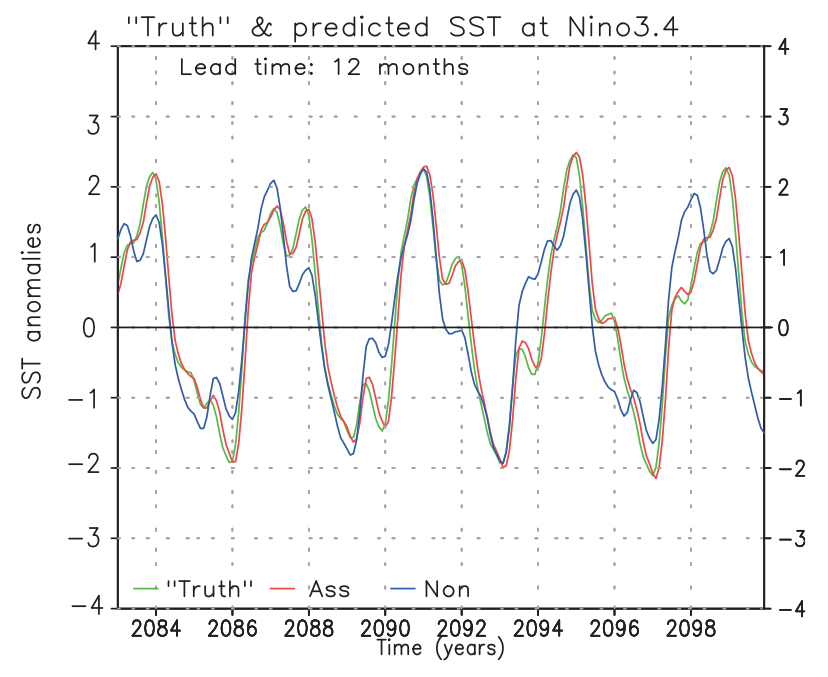

Fig. 10. Time series of the Niño3.4 indices for the "truth" value (green) and predictions made at 12-month lead times using initial conditions with (red) and without (blue) data assimilation during the model time period 2083/01 to 2099/12.

analysis. The prediction results with and without data assimilation are compared below.

Figure 10 presents the time series of the Niño3.4 indices for the "truth" value and predictions made at 12-month lead times using initial conditions with and without data as- similation during the model time period 2083/01-2099/12. The Niño3.4 indices in the assimilation case are very close to the "truth" value, whereas those in the non-assimilation case depart to a certain extent from the "truth" value. The correlation coefficient between the "truth" and the predicted Niño3.4 index in the assimilation case is 0.99 , whereas that between the "truth" and the predicted Niño3.4 index in the non-assimilation case is 0.84 . The RMSEs of the predicted Niño3.4 index for the assimilation and non-assimilation cases are 0.05 and 0.66 in the 1 -year lead time. The results are likely idealized to a certain extent because they are evaluated in a twin experiment, but these experiments provide us with important information about the way the 4D-Var data assimilation approach can effectively improve the model state estimation and prediction of ENSO events using the ICM.

\section{Conclusion and discussion}

Data assimilation is an effective way to improve the accuracy of model simulations and analyses for weather and climate through an optimal combination of model solutions and observations. In particular, the advanced 4D-Var data assimilation method is more dynamically and mathematically consistent in constraining numerical models with observations to achieve the optimal initialization for ENSO analysis and prediction. In this study, we implement the 4D-Var method based on an improved ICM that has been routinely used for 
real-time ENSO prediction. The construction of the 4D-Var assimilation system includes the tangent linear model and adjoint model of the ICM and a minimization procedure. Strict testing justifies the accuracy of the adjoint model and the effectiveness of the 4D-Var in constraining dynamical models with observations.

The impacts of the optimal initialization produced by 4DVar on ENSO analysis and prediction are evaluated through a biased twin experiment. In this study, only "observations" of the SST anomaly are assimilated into the model to optimize the initial conditions. Results show that, compared with the non-assimilation case, the assimilation results are more consistent with the "truth" value, and the RMSEs of the anomalies for the SST, $\tau$, SL and $T_{e}$ fields are much smaller (especially for the SST and $\tau$ fields). Additionally, the prediction accuracy is improved by optimizing the initial conditions. The results obtained in this study provide some insight into the way in which ENSO prediction can be improved with the 4D-Var algorithm.

The work performed in this study is a first step towards improving real-time ENSO analysis and prediction by applying the 4D-Var algorithm in the ICM. Further modeling studies using the 4D-Var are underway. As noted above, the ICM has been successfully used for real-time ENSO prediction, whose result, now named IOCAS ICM, is collected and posted every month at IRI/Columbia University, a multimodel product for real-time ENSO monitoring and prediction (see the IRI website). In this application, however, no sophisticated data assimilation is applied in the ICM; instead, a simple initialization method is currently taken for the model forecast, as follows: The observed interannual SST anomalies are the only field used in the prediction initialization (Zhang et al., 2013). In real-time practice, experimental predictions are typically conducted near the middle of each month, when the monthly mean SST fields from the previous month and the weekly mean SST fields from the first week of the current month are available from NOAA's Environment Modeling Center (Reynolds et al., 2002), which can be obtained online from the IRI data library. Then, the observed SST anomalies are used to derive interannual $\tau_{\text {inter }}$ fields using the empirical $\tau$ model. The derived $\tau_{\text {inter }}$ fields are taken to force the ocean model to produce an initial ocean state for the first day of each month, from which predictions are made. Additionally, as part of the initialization procedure, the observed SST anomalies are directly inserted into the ICM when making predictions. Based on results from this paper, the 4D-Var method will be incorporated in the ICM for real-time ENSO predictions.

Additionally, even without data assimilation, the forecasts using the ICM show a fairly high level of skill (Fig. 10, blue line) because the ENSO events simulated are so regular. This is attributed to the fact that stochastic atmospheric wind forcing is not included in the ICM (Zhang et al., 2008). In a more realistic global coupled climate model, however, the forecast skill of Niño 3.4 SST initialized by the SST-nudging scheme is very limited (Kumar et al., 2014; Zhu et al., 2015). In the future, we plan to assess the impact of the 4D-Var data assimilation in a more realistic way by including stochastic atmospheric forcing in the ICM, whose effects on ENSO simulations were evaluated by Zhang et al. (2008).

Furthermore, the 4D-Var method can also be used to optimize the model parameters, as demonstrated by the ensemble Kalman filter (Wu et al., 2012, 2016). For example, the performance of the ICM is sensitive to $\alpha_{\tau}$ and $\alpha_{\mathrm{Te}}$ (Zhang et al., 2005a; 2008); we plan to use 4D-Var to optimally determine these two parameters to further improve the ENSO prediction skill. In addition, the oceanic subsurface state has a considerable effect on SST in the tropical Pacific; thus, in addition to assimilating the observed SST field, observed subsurface thermal fields need to be assimilated into the ICM. In addition to the assimilation of oceanic fields, that of atmospheric data can also be considered. Note that during the 4D-Var assimilation process (the forward and backward time integrations of the model and its adjoint model), $\tau$ anomalies are internally determined using its anomaly model from the corresponding SST anomalies. Thus, the ICM with the 4D-Var has already taken into account the coupling between the ocean and atmosphere. So, the observed $\tau$ anomaly field can be introduced into the 4D-Var assimilation processes in a fairly straightforward way (that is, the coupled data assimilation). Taking all these together, it can ultimately be expected that real-time ENSO forecasting using the ICM can be improved through optimal initialization and parameter optimization using the 4D-Var data assimilation method.

Acknowledgements. We would like to thank Mu MU, Shaoqing ZHANG, Guijun HAN, Xuefeng ZHANG, Fei ZHENG, Qiang WANG, and Hui XU for their comments. The authors wish to thank the two anonymous reviewers for their comments, which helped to improve the original manuscript. This research is supported by the National Natural Science Foundation of China (Grant Nos. 41490644, 41475101 and 41421005), the CAS Strategic Priority Project (the Western Pacific Ocean System; Project Nos. XDA11010105, XDA11020306 and XDA11010301), the NSFCShandong Joint Fund for Marine Science Research Centers (Grant No. U1406401), and the NSFC Innovative Group Grant (Project No. 41421005).

Open Access. This article is distributed under the terms of the Creative Commons Attribution 4.0 International License (http://creativecommons.org/licenses/by/4.0/), which permits unrestricted use, distribution, and reproduction in any medium, provided you give appropriate credit to the original author(s) and the source, provide a link to the Creative Commons license, and indicate if changes were made.

\section{REFERENCES}

Balmaseda, M. A., D. L. T. Anderson, and M. K. Davey, 1994: ENSO prediction using a dynamical ocean model coupled to statistical atmospheres. Tellus A, 46(4), 497-511.

Barnett, T. P., N. Graham, S. Pazan, W. White, M. Latif, and M. Flügel, 1993: ENSO and ENSO-related predictability. Part I: Prediction of equatorial Pacific sea surface temperature with 
a hybrid coupled ocean-atmosphere model. J. Climate, 6, $1545-1566$.

Bjerknes, J., 1969: Atmospheric teleconnections from the equatorial Pacific. Mon. Wea. Rev., 97, 163-172.

Cane, M. A., S. E. Zebiak, and S. C. Dolan, 1986: Experimental forecasts of El Niño. Nature, 321(6073), 827-832.

Chen, D., S. E .Zebiak, A. J. Busalacchi , and Cane, M. A., 1995: An improved procedure for El Niño forecasting: Implications for predictability. Science, 269, 1699-1702.

Derber, J., and A. Rosati, 1989: A global oceanic data assimilation system. J. Phys. Oceanogr., 19(9), 1333-1347.

Dommenget, D., and D. Stammer, 2004: Assessing ENSO simulations and predictions using adjoint ocean state estimation. $J$. Climate, 17(22), 4301-4315.

Evensen, G., 1994: Sequential data assimilation with a nonlinear quasi-geostrophic model using Monte Carlo methods to forecast error statistics. J. Geophys. Res., 99, 10 143-10 162.

Galanti, E., E. Tziperman, M. Harrison, A. Rosati, and Z. Sirkes, 2003: A study of ENSO prediction using a hybrid coupled model and the adjoint method for data assimilation. Mon. Wea. Rev., 131(11), 2748-2764.

Han, G. J., W. Li, Z. J. He, K. X. Liu, and J. R. Ma, 2006: Assimilated tidal results of tide gauge and TOPEX/POSEIDON data over the China seas using a variational adjoint approach with a nonlinear numerical model. Adv. Atmos. Sci., 23, 449-460, doi: 10.1007/s00376-006-0449-8.

Han, G. J., X. R. Wu, S. Q. Zhang, Z. Y. Liu, I. M. Navon, and W. Li, 2015: A study of coupling parameter estimation implemented by 4D-Var and EnKF with a simple coupled system. Advances in Meteorology, 2015, doi: 10.1155/2015/530764.

Houtekamer, P. L., and H. L. Mitchell, 1998: Data assimilation using an ensemble Kalman filter technique. Mon. Wea. Rev., 126(3), 796-811.

Kalnay, E., 2003: Atmospheric Modeling, Data Assimilation and Predictability. Cambridge University Press, 342pp.

Keenlyside, N., and R. Kleeman, 2002: Annual cycle of equatorial zonal currents in the Pacific. J. Geophys. Res., 107(C8), 8-1.

Keenlyside, N., M. Latif, M. Botzet, J. Jungclaus, and U. Schulzweida, 2005: A coupled method for initializing El Niño Southern Oscillation forecasts using sea surface temperature. Tellus A, 57(3), 340-356.

Kirtman, B. P., and S. E. Zebiak, 1997: ENSO simulation and prediction with a hybrid coupled model. Mon. Wea. Rev., 125(10), 2620-2641.

Kleeman, R., A. M. Moore, and N. R. Smith, 1995: Assimilation of subsurface thermal data into a simple ocean model for the initialization of an intermediate tropical coupled oceanatmosphere forecast model. Mon. Wea. Rev., 123, 3103-3114.

Klinker, E., F. Rabier, G. Kelly, and J. F. Mahfouf, 2000: The ECMWF operational implementation of four-dimensional variational assimilation. III: experimental results and diagnostics with operational configuration. Quart. J. Roy. Meteor. Soc., 126, 1191-1215.

Kumar, A., H. Wang, Y. Xue, and W. Q. Wang, 2014: How much of monthly subsurface temperature variability in the equatorial Pacific can be recovered by the specification of sea surface temperatures?. J. Climate, 27, 1559-1577.

Liu, D. C., and J. Nocedal, 1989: On the limited memory BFGS method for large scale optimization. Mathematical Programming, 45, 503-528.

McCreary, J. P., 1981: A linear stratified ocean model of the equatorial undercurrent. Philos. Trans. Roy. Soc. London, 298,
603-635.

McCreary, J. P., Jr., 1983: A model of tropical ocean-atmosphere interaction. Mon. Wea. Rev., 111(2), 370-387.

Mu, M., W.-S. Duan, D. Chen, and W. D. Yu. 2015: Target observations for improving initialization of high-impact oceanatmospheric environmental events forecasting. National Science Review, 2, 226-236.

Navon, I. M., X. Zou, J. Derber, and J. Sela, 1992: Variational data assimilation with an adiabatic version of the NMC spectral model. Mon. Wea. Rev., 120, 1433-1446.

Neelin, J. D., 1990: A hybrid coupled general circulation model for El Niño studies. J. Atmos. Sci., 47(5), 674-693.

Peng, S. Q., and L. Xie, 2006: Effect of determining initial conditions by four-dimensional variational data assimilation on storm surge forecasting. Ocean Modelling, 14(1), 1-18.

Philander, S. G. H., R. C. Pacanowski, N. C. Lau, and M. J. Nath, 1992: Simulation of ENSO with a global atmospheric GCM coupled to a high-resolution tropical Pacific Ocean GCM. J. Climate, 5(4), 308-329.

Reynolds, R. W., N. A. Rayner, T. M. Smith, D. C. Stokes, and W. Wang, 2002: An improved in situ and satellite SST analysis for climate. J. Climate, 15(13), 1609-1625.

Rosati, A., K. Miyakoda, and R. Gudgel, 1997: The impact of ocean initial conditions on ENSO forecasting with a coupled model, Mon. Wea. Rev., 125(5), 754-772.

Sugiura, N., T. Awaji, S. Masuda, T. Mochizuki, T. Toyoda, T. Miyama, H. Igarashi, and Y. Ishikawa, 2008: Development of a four-dimensional variational coupled data assimilation system for enhanced analysis and prediction of seasonal to interannual climate variations. J. Geophys. Res., 113(C10), C10017.

Tang, Y. M., and W. W. Hsieh, 2001: Coupling neural networks to incomplete dynamical systems via variational data assimilation. Mon. Wea. Rev., 129(4), 818-834.

Tang, Y. M., J. Ambandan, and D. K. Chen, 2014: Nonlinear measurement function in the ensemble Kalman filter. Adv Atmos. Sci., 31(3), 551-558, doi: 10.1007/s00376-013-3117-9.

Wang, B., X. L. Zou, and J. Zhu, 2000: Data assimilation and its applications. Proceedings of the National Academy of Sciences of the United States of America, 97(21), 11 143-11 144.

Weaver, A. T., J. Vialard, and D. L. T. Anderson, 2003: Three and four dimensional variational assimilation with a general circulation model of the tropical Pacific Ocean: Part I: formulation, internal diagnostics, and consistency checks. Mon. Wea. Rev., 131, 1360-1378.

Wu, X. R., S. Q. Zhang, Z. Y. Liu, A. Rosati, T. L. Delworth, and Y. Liu, 2012: Impact of geographic-dependent parameter optimization on climate estimation and prediction: Simulation with an intermediate coupled model. Mon. Wea. Rev., 140(12), 3956-3971.

Wu, X. R., W. Li, G. J. Han, S. Q. Zhang, and X. D. Wang, 2014: A compensatory approach of the fixed localization in EnKF. Mon. Wea. Rev., 142, 3713-3733.

Wu, X. R., G. J. Han, S. Q. Zhang, and Z. Y. Liu, 2016: A study of the impact of parameter optimization on ENSO predictability with an intermediate coupled model. Climate Dyn., 46, 711727, doi: 10.1007/s00382-015-2608-z.

Wyrtki, K., 1975: El Niño-the dynamic response of the equatorial Pacific Ocean to atmospheric forcing. J. Phys. Oceanogr., 5(4), 572-584.

Zebiak, S. E., and M. A. Cane, 1987: A model El Niño-Southern oscillation. Mon. Wea. Rev., 115, 2262-2278. 
Zhang, R. H., and C. Gao, 2015: Role of subsurface entrainment temperature (Te) in the onset of El Niño events, as represented in an intermediate coupled model. Climate Dyn., 1-19, doi: 10.1007/s00382-015-2655-5.

Zhang, R. H., S. E. Zebiak, R. Kleeman, and N. Keenlyside, 2003: A new intermediate coupled model for El Niño simulation and prediction. Geophys. Res. Lett., 30(19), doi:10.1029/2003 GL018010, 19.

Zhang, R. H., R. Kleeman, S. E. Zebiak, N. Keenlyside, and S. Raynaud, 2005a: An empirical parameterization of subsurface entrainment temperature for improved SST anomaly simulations in an intermediate ocean model. J. Climate, 18, 350371.

Zhang, R. H., S. E. Zebiak, R. Kleeman, and N. Keenlyside, 2005b: Retrospective El Niño forecasts using an improved intermediate coupled model. Mon. Wea. Rev., 133, 2777-2802.

Zhang, R. H., A. J. Busalacchi, and D. G. DeWitt, 2008: The roles of atmospheric stochastic forcing $(S F)$ and oceanic entrainment temperature $\left(T_{e}\right)$ in decadal modulation of ENSO. $J$. Climate, 21, 674-704.

Zhang, R. H., F. Zheng, J. Zhu, and Z. G. Wang, 2013: A successful real-time forecast of the 2010-11 La Niña event. Sci. Rep., 3, 1108, doi: 10.1038/srep01108.

Zhang, R. H., C. Gao, X. B. Kang, H. Zhi, Z. G. Wang, and L. C. Feng, 2015: ENSO modulations due to interannual variability of freshwater forcing and ocean biology-induced heating in the tropical Pacific. Sci. Rep.., 5, 18506, doi: 10.1038/srep18506.

Zhang, S., X. Zou, and J. E. Ahlquist, 2001: Examination of numerical results from tangent linear and adjoint of discontinuous nonlinear models. Mon. Wea. Rev., 129(11), 2791-2804.

Zhang, S., M. J. Harrison, A. T. Wittenberg, A. Rosati, J. L. Anderson, and V. Balaji, 2005c: Initialization of an ENSO forecast system using a parallelized ensemble filter. Mon. Wea. Rev., 133(11), 3176-3201.
Zhang, S., M. J. Harrison, A. Rosati, and A. Wittenberg, 2007: System design and evaluation of coupled ensemble data assimilation for global oceanic climate studies. Mon. Wea. Rev., 135(10), 3541-3564.

Zhang, S., Y. S. Chang, X. Yang, and A. Rosati, 2014: Balanced and coherent climate estimation by combining data with a biased coupled model. J. Climate, 27(3), 1302-1314.

Zhang, X. F., S. Q. Zhang, Z. Y. Liu, X. R. Wu, and G. J. Han, 2015a: Parameter optimization in an intermediate coupled climate model with biased physics. J. Climate, 28(3), 1227 1247.

Zhang, X. F., G. J. Han, D. Li, X. R. Wu, W. Li, and P. C. Chu, 2015b: Variational estimation of wave-affected parameters in a two-equation turbulence model. J. Atmos. Oceanic Technol., 32(3), 528-546.

Zheng, F., J. Zhu, R. H. Zhang, and G. Q. Zhou, 2006: Ensemble hindcasts of SST anomalies in the tropical Pacific using an intermediate coupled model. Geophys. Res. Lett., 33(19), L19604.

Zheng, F., J. Zhu, H. Wang, and R. H. Zhang, 2009: Ensemble hindcasts of ENSO events over the past 120 years using a large number of ensembles. Adv. Atmos. Sci., 26, 359-372, doi: 10.1007/s00376-009-0359-7.

Zhu, J., G. Q. Zhou, C. X. Yan, W. W. Fu, and X. B. You, 2006: A three-dimensional variational ocean data assimilation system: scheme and preliminary results. Science in China Series D: Earth Sciences, 49(11), 1212-1222.

Zhu, J. S., A. Kumar, H. Wang, and B. H. Huang, 2015: Sea surface temperature predictions in NCEP CFSv2 using a simple ocean initialization scheme. Mon. Wea. Rev., 143, 31763191.

Zou, X., I. M. Navon, M. Berger, K. H. Phua, T. Schlick, and F. X. Le Dimet, 1993: Numerical experience with limited-memory quasi-Newton and truncated Newton methods. SIAM Journal on Optimization, 3(3), 582-608. 